\title{
Magnetoresistance and transistor-like behavior of a double quantum-dot via crossed Andreev reflections
}

\author{
E. C. Siqueira ${ }^{1}$, 国 and G. G. Cabrera 2 , b) \\ 1) Departamento de Física e Química, \\ Universidade Estadual Paulista - UNESP, \\ Av. Brasil, Centro, 15385-000 - Ilha Solteira, SP - Brasil \\ ${ }^{2)}$ Instituto de Física 'Gleb Wataghin', Universidade Estadual de Campinas (UNICAMP), \\ Campinas 13083-859, SP, Brasil
}

(Dated: 11 November 2018)

The electric current and the magnetoresistance effect are studied in a double quantum-dot system, where one of the dots $Q D_{a}$ is coupled to two ferromagnetic electrodes $\left(F_{1}, F_{2}\right)$, while the second $Q D_{b}$ is connected to a superconductor $S$. For energy scales within the superconductor gap, electric conduction is allowed by Andreev reflection processes. Due to the presence of two ferromagnetic leads, non-local crossed Andreev reflections are possible. We found that the magnetoresistance sign can be changed by tuning the external potential applied to the ferromagnets. In addition, it is possible to control the current of the first ferromagnet $\left(F_{1}\right)$ through the potential applied to the second one $\left(F_{2}\right)$. We have also included intradot interaction and gate voltages at each quantum dot and analyzed their influence through a mean field approximation. The interaction reduces the current amplitudes with respect to the non-interacting case, but the switching effect still remains as a manifestation of quantum coherence, in scales of the order of the superconductor coherence length.

PACS numbers: $73.23 \mathrm{Hk}, 73.63 \mathrm{Kv}, 74.45 .+\mathrm{c}, 74.78 \mathrm{Na}$

\section{INTRODUCTION}

The study of transport properties of hybrid nanostructures is a very active field of research, involving new and interesting physical phenomena that appear at the nanometer scale, with great potential for developing future technology in mesoscopic systems. Within this context, systems based on combinations of superconductors and ferromagnets are particularly interesting, since the interplay between these two phenomena can give rise to unusual effects. It is well known that in ferromagnetic/superconducting $(F / S)$ junctions the conductance can be controlled through the ferromagnet polarization. For energies within the superconductor gap, the conduction process is established via Andreev reflections 1 (AR). In this process, two electrons of $F$ with opposite spins recombine into a Cooper pair in $S$ (with total spin $S=0$ ). The Cooper pairs are the supercurrent carriers of the superconductor and these pairs are highly correlated in large distances in comparison to the interatomic distances. This feature has been explored by Deutscher and Feinberg ${ }^{2}$ to propose a non-local Andreev reflection (called crossed AR), where two electrons of different leads can combine into a Cooper pair if the distance between these leads is smaller than the coherence length. Since this proposal, there has been a profusion of works exploring crossed AR in different geometries ${ }^{3}-11$, resonant nanostructures involving quantum $\operatorname{dots}^{12}-17$, different conduction regimes ${ }^{18,19}$ (ballistic and diffusive), and addressing more fundamental

\footnotetext{
a) Electronic mail: ecosta@dfq.feis.unesp.br

b) Electronic mail: cabrera@ifi.unicamp.br
}

questions, e.g., the entanglement of the quasiparticles in different leads ${ }^{20-25}$. Within this vast set of hybrid nanostructures, systems composed by double quantum dots (QDs) are very promising, since this association can serve as a model of diatomic molecules ${ }^{26-28}$. Many works involving double QDs have been developed mainly concerning the Kondo effect ${ }^{29}-31$, scattering with spin inversion ${ }^{32}$, effects of different geometries ${ }^{33.34}$ (series and parallel association), spin detectors 35,36 and systems involving superconductors. In the latter case, there are studies involving Josephson molecular junctions ${ }^{37-39}$ and transport by $\mathrm{AR}^{40,41}$.

By considering the outstanding properties of crossed ARs and the promising feature of double QDs, we propose a prototype of a molecular transistor by combining two QDs with a superconductor and two ferromagnetic electrodes. A schematic diagram of the system is shown in Fig. 1. There are two ferromagnetic electrodes, $F_{1}$ and $F_{2}$, attached to the first QD and a superconductor electrode is connected to the second one. The dot coupled to the ferromagnetic electrodes $(F)$ is called $a$, and $b$ is the one coupled to the superconductor $(S)$. The superconductor has its chemical potential fixed to zero, and independent voltage bias are applied to the ferromagnets which are called $V_{1}$ and $V_{2}$. There are also gate potentials applied to the dots, denoted by $V_{g a}$ and $V_{g b}$. By exploring the resonant structure of the local density of states (LDOS) and the non-local feature of the crossed AR, we show that is possible to switch the current at one ferromagnetic lead by the applied bias in the second one. In addition, the magnetoresistance sign can also be changed through the bias. The control of the current via external parameters can be of interest in applications of molecular electronics. 
We assume the existence of an intradot interaction at each QD and use a mean-field approximation to include its effect in our calculation. However, we have not considered the occurrence of Kondo resonances at the QD's. While the Kondo effect has been experimentally observed in semiconducting QDs, coupling the dot to a ferromagnetic electrode will split the dot level, leading to the suppression of the Kondo effect ${ }^{42,43}$. Electron pairing in the superconductor electrode also competes with Kondo through the proximity effect $\underline{44}$. Now, a discussion about the relative magnitude of the correlation parameters is in order. In this paper, $\mathcal{U}$ is limited to the gap value, since we analyzed the contribution of a pure Andreev current (subgap current). Our study is then confined to the weak correlation regime. This also restricts the voltages to very small values, typically of the order of $\mathrm{mV}$ or smaller. In fact, in the experiment by Beckmann $\frac{45}{2}$ et. $a l$. , the superconductor gap of the $A l$ film with thickness of $80 \mathrm{~nm}$ was found to be $\sim 0.18 \mathrm{meV}$. In another experiment performed by Russo $\underline{\underline{46}}$ et. al., using $N b$ films, the superconductor gap obtained was in the rage of 0.90 and $1.45 \mathrm{meV}$, for films with thicknesses between 15 and $50 \mathrm{~nm}$, respectively.

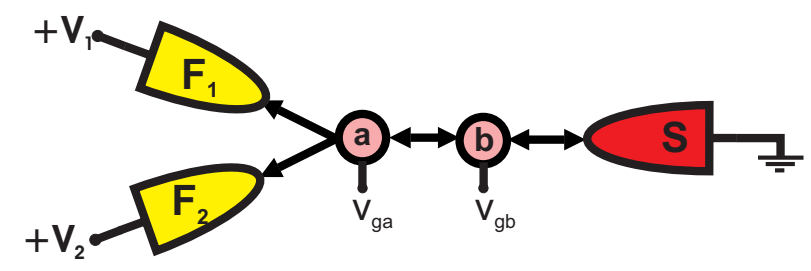

FIG. 1. (Color Online) Schematic diagram showing the $\left(F_{1}, F_{2}\right)-Q D_{a}-Q D_{b}-S$ system. The magnetization of $F_{1}$ is assumed to be fixed and the magnetization of $F_{2}$ can be varied for an angle $\theta$ with respect to the $F_{1}$ magnetization. $V_{1}$ and $V_{2}$ are the external potentials applied to $F_{1}$ and $F_{2}$, respectively while the superconductor is grounded. Gate voltages are also applied to the QDs, with $V_{g a}$ and $V_{g b}$ being the potentials applied to $a$ and $b$, respectively.

\section{MODEL AND FORMULAS}

The system displayed in Fig. 1 is described by the following Hamiltonian:

$$
\mathcal{H}=\mathcal{H}_{1}+\mathcal{H}_{2}+\mathcal{H}_{S}+\mathcal{H}_{d q d}+\mathcal{H}_{T}
$$

where the ferromagnet $F_{1}$ is modeled by the Stoner model $\mathbb{l 7}^{47}$ given by

$$
\mathcal{H}_{1}=\sum_{k \sigma} \epsilon_{1 k \sigma} \hat{a}_{k \sigma}^{\dagger} \hat{a}_{k \sigma}
$$

with $\epsilon_{1 k \sigma}=\epsilon_{k}-\operatorname{sgn}(\sigma) h_{1}-\mu_{1}$. In the same way, the lead $F_{2}$ is described by

$$
\mathcal{H}_{2}=\sum_{k \sigma} \epsilon_{2 k \sigma} \hat{b}_{k \sigma}^{\dagger} \hat{b}_{k \sigma}-\sum_{k \sigma} h_{2} \sin \theta \hat{b}_{k \sigma}^{\dagger} \hat{b}_{k \bar{\sigma}}
$$

with $\epsilon_{2 k \sigma}=\epsilon_{k}-\operatorname{sgn}(\sigma) h_{2} \cos \theta-\mu_{2}$.

The spin bands of $F_{1}\left(F_{2}\right)$ are split by the exchange energy $h_{1}\left(h_{2}\right)$ and the magnetization direction of $F_{2}$ has an angle $\theta$ with respect to the magnetization of $F_{1}$. By changing the value of $\theta$, we can change the configuration of the system from parallel alignment $(\theta=0)$ to an antiparallel alignment $(\theta=\pi)$.

The superconductor is described by the BCS Hamiltonian ${ }^{48}$,

$$
\mathcal{H}_{S}=\sum_{k \sigma} \epsilon_{k} s_{k \sigma}^{\dagger} \hat{s}_{k \sigma}+\sum_{k}\left[\Delta \hat{s}_{k \uparrow}^{\dagger} \hat{s}_{-k \downarrow}^{\dagger}+\text { H.c. }\right]
$$

with $\Delta$ being the superconductor gap and the operator $\hat{s}_{k \uparrow}^{\dagger} \hat{s}_{-k \downarrow}^{\dagger}$ creates a Cooper pair in $S$. Therefore, we are considering here a conventional singlet superconductor with $s$-wave pairing symmetry.

The chemical potentials $\mu_{1}$ and $\mu_{2}$ of $F_{1}$ and $F_{2}$ are fixed by the applied bias $V_{1}$ and $V_{2}$ while the superconductor chemical potential $\left(\mu_{S}\right)$ is set to zero as the ground.

The QDs are modeled by the mean-field Hamiltonian,

$$
\mathcal{H}_{d q d}=\sum_{\sigma} E_{a \sigma} \hat{n}_{a \sigma}+\sum_{\sigma} E_{b \sigma} \hat{n}_{b \sigma}
$$

where $E_{a \sigma}=E_{a}-e V_{g a}+\mathcal{U}\left\langle\hat{n}_{a \bar{\sigma}}\right\rangle / 2$ and $E_{b \sigma}=E_{b}-$ $e V_{g b}+\mathcal{U}\left\langle\hat{n}_{b \bar{\sigma}}\right\rangle / 2$. The QDs energy levels, $E_{a \sigma}$ and $E_{b \sigma}$, are renormalized by the intradot interaction $\mathcal{U}$. This interaction couples the energy levels to the mean occupations $\left\langle\hat{n}_{a \bar{\sigma}}\right\rangle$ and $\left\langle\hat{n}_{b \bar{\sigma}}\right\rangle$. In addition, gate voltages $V_{g a}$ and $V_{g b}$ allow one to tune the position of the bare QD levels with respect to superconductor chemical potential.

The tunneling between the QDs and leads is described by

$$
\begin{gathered}
\mathcal{H}_{T}=\sum_{k \sigma}\left[t_{1} \hat{a}_{k \sigma}^{\dagger} \hat{c}_{a \sigma}+\text { H.c. }\right]+\sum_{k \sigma}\left[t_{2} \hat{b}_{k \sigma}^{\dagger} \hat{c}_{a \sigma}+\text { H.c. }\right] \\
+\sum_{k \sigma}\left[t_{s} \hat{s}_{k \sigma}^{\dagger} \hat{c}_{b \sigma}+\text { H.c. }\right]+\sum_{\sigma}\left[t_{a b} \hat{c}_{a \sigma}^{\dagger} \hat{c}_{b \sigma}+\text { H.c. }\right]
\end{gathered}
$$

where the last term accounts for the hopping between the QD's. For simplicity, we have assumed that the hopping matrix elements are independent of the spin index. This will safely cover the case of homogeneous 'monodomain' ferromagnets, with no spin-flip scattering. Study of more general situations for the $S / F$ interface shows the possibility of inducing a 'triplet proximity effect' in the ferromagnet from scattering by inhomogeneities at the interface or at domain walls in the ferromagnet $\underline{49}-55$. In this latter case, anomalous Andreev reflections, i.e. reflections with spin-flip into the triplet state, have to be considered as also contributing to the current. In the present study, the above phenomenon will not be taken into account, restricting our calculation to ideal homogeneous leads. Note that in real experimental setups, small magnetic fields can remove any domain structure.

In order to calculate the transport properties we have used the non-equilibrium Green's function method ${ }^{56}$. 
All the physical quantities can be cast in terms of the Green's function of the QD's. Since we are dealing with ferromagnet and superconductor order parameters, it is convenient to introduce the Nambu representation by using a generalized four-dimensional spinor $\hat{\mathbf{\Psi}}_{i}=$ $\left(\begin{array}{llll}\hat{c}_{i \uparrow}^{\dagger} & \hat{c}_{i \downarrow} & \hat{c}_{i \downarrow}^{\dagger} & \hat{c}_{i \uparrow}\end{array}\right)^{\dagger}$ with $i=a, b$. This allows one to treat both order parameters on the same footing.

In terms of Nambu spinors the lesser $\left(\mathbf{G}^{<}\right)$and retarded/advanced Green function $\mathbf{G}^{r / a}$ of QDs are written as

$$
\mathbf{G}_{i i}^{<}\left(t_{1}, t_{2}\right)=i\left\langle\hat{\mathbf{\Psi}}_{i}\left(t_{1}\right) \otimes \hat{\mathbf{\Psi}}_{i}^{\dagger}\left(t_{2}\right)\right\rangle
$$

and

$$
\begin{aligned}
\mathbf{G}_{i i}^{r / a}\left(t_{1}, t_{2}\right)=\mp i \vartheta\left( \pm t_{1} \mp t_{2}\right)\left\langle\hat{\mathbf{\Psi}}_{i}\left(t_{1}\right) \otimes \hat{\mathbf{\Psi}}_{i}^{\dagger}\left(t_{2}\right)\right. \\
\left.+\hat{\mathbf{\Psi}}_{i}^{\dagger}\left(t_{2}\right) \otimes \hat{\mathbf{\Psi}}_{i}\left(t_{1}\right)\right\rangle
\end{aligned}
$$

with $i=a, b$. Similiar definitions are given for the leads Green functions. However, all the physical quantities are determined from the Green functions of the QDs.

Under stationary regime, the current through the system is time-independent and we can work with the Fourier transform of the Green functions. In this case, the total electrical current coming from the ferromagnets and injected into the superconductor is given by

$I=\frac{e}{h} \int d \omega\left[\mathbf{G}_{a a}^{r}(\omega) \boldsymbol{\Sigma}_{F}^{<}(\omega)+\mathbf{G}_{a a}^{<}(\omega) \boldsymbol{\Sigma}_{F}^{a}(\omega)+\text { H.c. }\right]_{11+33}$.

The subscript " $11+33$ " means taking the sum of 11 and 33 elements of the $4 \times 4$ matrix. By adopting the equation of motion method, the Green function of the dot $a$ has been determined:

$$
\mathbf{G}_{a a}^{r}=\mathbf{G}_{a a}^{r 0}+\mathbf{G}_{a a}^{r} \mathbf{t}_{a b}^{\dagger} \mathbf{G}_{b b}^{r 0} \mathbf{t}_{a b} \mathbf{G}_{a a}^{r 0},
$$

with $\mathbf{G}_{a a}^{r 0}=\mathbf{g}_{a a}^{r}\left(\mathbf{1}-\boldsymbol{\Sigma}_{F}^{r} \mathbf{g}_{a a}^{r}\right)^{-1}$ and $\mathbf{G}_{b b}^{r 0}=\mathbf{g}_{b b}^{r}(\mathbf{1}-$ $\left.\mathbf{\Sigma}_{S}^{r} \mathbf{g}_{b b}^{r}\right)^{-1}$.

In these equations $\mathbf{G}_{a a}^{r}$ is the Green's function of the quantum dot $a ; \mathbf{G}_{b b}^{r}$ is the Green's function of the quantum dot $b ; \mathbf{g}_{a a}^{r}$ and $\mathbf{g}_{b b}^{r}$ are the Green's functions of the dots $a$ and $b$ isolated from the electrodes; $\mathbf{t}_{a b}$ describes the coupling between the dots; $\boldsymbol{\Sigma}_{F}^{r}=\boldsymbol{\Sigma}_{1}^{r}+\boldsymbol{\Sigma}_{2}^{r}$ and $\boldsymbol{\Sigma}_{S}^{r}$ are the retarded self-energies describing the coupling of the dots with the ferromagnetic and superconductor electrodes, respectively. Explicitly, these self-energies are written as,

$$
\boldsymbol{\Sigma}_{F}^{r, a}(\omega)=\mp \frac{i}{2}\left[\begin{array}{cccc}
A_{\uparrow} & 0 & B & 0 \\
0 & A_{\downarrow} & 0 & B \\
B & 0 & A_{\downarrow} & 0 \\
0 & B & 0 & A_{\uparrow}
\end{array}\right],
$$

with $A_{\sigma} \equiv \Gamma_{1 \sigma}+c^{2} \Gamma_{2 \sigma}+s^{2} \Gamma_{2 \bar{\sigma}}, B=s c\left(\Gamma_{2 \uparrow}-\Gamma_{2 \downarrow}\right)$, $s \equiv \sin \theta / 2$ and $c \equiv \cos \theta / 2$. We also have defined $\Gamma_{i \sigma}=$ $2 \pi\left|t_{i}\right|^{2} N_{i \sigma}$, (with $i=1,2$ ) as the coupling strength, with $t_{i}$ being the tunneling amplitude and $N_{i \sigma}$ the density of states for the ferromagnet spin $\sigma$ band.
The retarded/advanced self-energy of the superconductor is given by,

$$
\boldsymbol{\Sigma}_{S}^{r, a}(\omega)=\mp \frac{i}{2} \Gamma_{s} \rho(\omega)\left[\begin{array}{cccc}
1 & -\Delta / \omega & 0 & 0 \\
-\Delta / \omega & 1 & 0 & 0 \\
0 & 0 & 1 & \Delta / \omega \\
0 & 0 & \Delta / \omega & 1
\end{array}\right]
$$

where $\Gamma_{s}=2 \pi\left|t_{s}\right|^{2} N_{s}$, with $N_{s}$ being the density of states of the superconductor in the normal state and $\rho$ is the modified BCS density of states

$$
\rho(\omega) \equiv \frac{|\omega| \vartheta(|\omega|-\Delta)}{\sqrt{\omega^{2}-\Delta^{2}}}+\frac{\omega \vartheta(\Delta-|\omega|)}{i \sqrt{\Delta^{2}-\omega^{2}}}
$$

with the imaginary part accounting for Andreev states within the gap 57 .

It is important to note that the definition of $\hat{\mathbf{\Psi}}_{i}$ is the same as the one used in Refs. 57 59. As a result, the self-energies given by Eqs. (6) and (7) are the same as those found in Refs. 57 -59.

The "lesser" Green's function is obtained through the Keldysh equation

$$
\mathbf{G}_{a a}^{<}(\omega)=\mathbf{G}_{a a}^{r}(\omega) \mathbf{\Sigma}_{T a}^{<}(\omega) \mathbf{G}_{a a}^{a}(\omega)
$$

with $\boldsymbol{\Sigma}_{T a}^{<}(\omega)=\boldsymbol{\Sigma}_{F}^{<}(\omega)+\mathbf{t}_{a b}^{\dagger} \mathbf{G}_{b b}^{r 0} \boldsymbol{\Sigma}_{S}^{<}(\omega) \mathbf{G}_{b b}^{a 0}(\omega) \mathbf{t}_{a b}$.

The self-energies $\boldsymbol{\Sigma}_{F}^{<}=\boldsymbol{\Sigma}_{1}^{<}+\boldsymbol{\Sigma}_{2}^{<}$and $\boldsymbol{\Sigma}_{S}^{<}$are obtained by the fluctuation-dissipation theorem $\boldsymbol{\Sigma}_{i}^{<}=\mathbf{F}_{i}(\omega)\left[\boldsymbol{\Sigma}_{i}^{a}-\right.$ $\boldsymbol{\Sigma}_{i}^{r}$, where $i=1,2$ or $s$. The Fermi matrix $\mathbf{F}_{i}$ is given by,

$$
\mathbf{F}_{i}(\omega)=\left[\begin{array}{cccc}
f_{i} & 0 & 0 & 0 \\
0 & \bar{f}_{i} & 0 & 0 \\
0 & 0 & f_{i} & 0 \\
0 & 0 & 0 & \bar{f}_{i}
\end{array}\right]
$$

in which the Fermi functions are defined as $f_{i}=f(\omega-$ $\left.e V_{i}\right)$ and $\bar{f}_{i}=f\left(\omega+e V_{i}\right)$ for $i=1,2$ and $f_{i}=f(\omega)$, if $i=s$.

Since the Green's functions are dependent on mean occupations through the intradot interaction, it is necessary to calculate those quantities at the dots. From the definition of the "lesser" Green's function, one straightforwardly obtains the system of equations below:

$$
\begin{aligned}
& \left\langle n_{a \uparrow}\right\rangle=\frac{1}{2 \pi i} \int_{-\infty}^{+\infty} G_{a a, 11}^{<}\left[\omega,\left\langle n_{a \uparrow}\right\rangle,\left\langle n_{a \downarrow}\right\rangle,\left\langle n_{b \uparrow}\right\rangle,\left\langle n_{b \downarrow}\right\rangle\right] \\
& \left\langle n_{a \downarrow}\right\rangle=\frac{1}{2 \pi i} \int_{-\infty}^{+\infty} G_{a a, 33}^{<}\left[\omega,\left\langle n_{a \uparrow}\right\rangle,\left\langle n_{a \downarrow}\right\rangle,\left\langle n_{b \uparrow}\right\rangle,\left\langle n_{b \downarrow}\right\rangle\right] \\
& \left\langle n_{b \uparrow}\right\rangle=\frac{1}{2 \pi i} \int_{-\infty}^{+\infty} G_{b b, 11}^{<}\left[\omega,\left\langle n_{a \uparrow}\right\rangle,\left\langle n_{a \downarrow}\right\rangle,\left\langle n_{b \uparrow}\right\rangle,\left\langle n_{b \downarrow}\right\rangle\right] \\
& \left\langle n_{b \downarrow}\right\rangle=\frac{1}{2 \pi i} \int_{-\infty}^{+\infty} G_{b b, 33}^{<}\left[\omega,\left\langle n_{a \uparrow}\right\rangle,\left\langle n_{a \downarrow}\right\rangle,\left\langle n_{b \uparrow}\right\rangle,\left\langle n_{b \downarrow}\right\rangle\right]
\end{aligned}
$$

These integral equations must be solved numerically in a self-consistent way. Once the occupation numbers 
are obtained, it is possible to calculate the other physical quantities.

By using the relations above, it is possible to determine the electrical current as a function of the applied bias $V_{1}$ and $V_{2}$, the magnetization angle $\theta$ and the gate potentials, $V_{g a}$ and $V_{g b}$. The total current is obtained by adding the two currents from both ferromagnets. These currents are summed in the QDs and injected into the superconductor by means of the Andreev reflection. In this process, an incident electron coming from the ferromagnetic lead, with energy $\omega$ and spin $\sigma$, combines with a second electron with energy $-\omega$ and spin $-\sigma$. Both electrons enter the superconductor as a Cooper pair, leaving a reflecting hole with spin $-\sigma$ in the ferromagnetic electrode. Since we need both spins to create a Cooper pair, the AR is prohibited when the polarization of the ferromagnetic lead is equal to unity. In the setup under consideration (see Fig. 1), the Andreev reflection may be local, that is occurring in the same lead of the incident electron, or may be nonlocal, with the reflected hole appearing in the other lead. For instance, an incident electron in $F_{1}$ can be reflected as a hole at $F_{1}$ or $F_{2}$. The latter case, called crossed AR, is possible only if the distance between $F_{1}$ and $F_{2}$ is of the order of or less than the superconductor coherence length. Recent experiments probing crossed ARs, estimate superconducting coherence lengths in the range of 10-15 nm for $\mathrm{Nb}$ films 46 , and $200-300 \mathrm{~nm}$ for $\mathrm{Al}$ films $\frac{45}{}$, depending on the sample, but showing that the effect can be checked experimentally within the present state of the art in nanodevices. Crossed ARs allow us to control the current through the angle $\theta$ and the polarization of the ferromagnets. The polarization is defined in terms of the coupling constants:

$$
P_{i}=\frac{\Gamma_{i \uparrow}-\Gamma_{i \downarrow}}{\Gamma_{i \uparrow}+\Gamma_{i \downarrow}}, \quad i=1,2 .
$$

The most interesting case is the one when both leads are full polarized. In this case, AR in the same electrode is not possible and the crossed AR is the only mechanism to carry current through the system ${ }^{60}$. As a result, the current can be tuned from zero to its maximum by varying the angle $\theta$ of the magnetization of $F_{2}$. In fact, when $\theta=0$, the total current is zero since we have the same spin in both electrodes which implies no availability of states for the reflected hole. On the other hand, when $\theta=\pi$, all the electrons of $F_{1}$ are up-spin and the electrons of $F_{2}$ are down-spin and the current exhibits a maximum value. Materials with a high degree of spin polarization are currently being used to study spin-dependent transport properties. The most promising case corresponds to $\mathrm{CrO}_{2}$, which has been predicted to be half-metallic and $100 \%$ polarized at the Fermi level ${ }^{61}$.

In order to compare the current in these two different configurations, we define the Andreev magnetoresistance as:

$$
A R M R=\frac{\left|I_{A P}\right|-\left|I_{P}\right|}{\left|I_{A P}\right|+\left|I_{P}\right|}
$$

in which $I_{A P}=I(\theta=\pi)$ and $I_{P}=I(\theta=0)$.

The definition (11) is different from the usual one, since we use the absolute value of the currents. This definition allows us to compare the amplitude of the currents in terms of the bias of each electrode. In this system the sign of current in each ferromagnetic terminal is linked to the averaged chemical potential of the two leads. Thus, it contains the case that $V_{1}>0$ and $V_{2}<0$ but $I>$ 0 . This unusual behavior is characteristic of the crossed AR reflection and has been first pointed out by Y. Zhu et al. in a one-dot three-terminal system ${ }^{58}$. By using the definition (11) we can determine which current is larger through the sign of $A R M R$, even in cases when we consider the dependence of $A R M R$ with the bias $V_{1}$ or $V_{2}$.

\section{RESULTS AND DISCUSSION}

Some $A R M R$ curves are presented in Fig. $2 \mathrm{a}$ for different values of the applied bias in the electrodes $F_{1}$ and $F_{2}$. For $V_{2}=0, A R M R$ is positive in the entire range of $V_{1}$ with a rapid oscillation around $V_{1}=0$. For $V_{2}=+0.30$ the $A R M R$ displays a step-like behavior with positive values for $V_{1}>0$ and negative values for $V_{1}<0$. The trend is inverted for $V_{2}=-0.30$. These results indicate that one can control the sign of the system magnetoresistance through external parameters $V_{1}$ and $V_{2}$. In order to understand the $A R M R$ curves, in Figs. $2 \mathrm{~b}$ and $2 \mathrm{r}$ the corresponding $I_{P}$ and $I_{A P}$ curves are shown. In the parallel configuration, the total current $I_{P}$ is very small since the polarization values $\left(P_{1}=P_{2}=0.95\right)$ are close to unity. In this case, the crossed AR does not contribute significantly since the magnetizations of $F_{1}$ and $F_{2}$ are pointing in the same direction. When the magnetization of $F_{2}$ is inverted, the crossed AR dominates the conduction process making $I_{A P}$ much higher than $I_{P}$. In this way, when the polarization is close to unity, the usual situation is to find positive values of $A R M R$ (see Eq. (11)) since the current $I_{A P}$ is mainly carried by the crossed AR plus a small direct AR contribution. However, as shown in Fig. $2 \mathrm{a}$, for $V_{2} \neq 0$, the $A R M R$ presents negative values even for high values of the $P_{1}$ and $P_{2}$. In fact, the potential $V_{2}$ shifts $I_{P}$ and $I_{A P}$ along the current axis, as shown in Fig. 2b. In this case, the current $I_{P}$ can be higher than $I_{A P}$ for some range of $V_{1}$ even if the amplitude of the former is close to zero.

The parameters controlling the amplitude and the shift of the currents with $V_{1}$ and $V_{2}$ are the coupling constants $\Gamma_{1}$ and $\Gamma_{2}$, respectively. In fact, by increasing $\Gamma_{1}$ and $\Gamma_{2}$, the admixture of states at the ferromagnets with the dot levels is also increased. Therefore, more electrons can be transferred to the superconductor by direct ARs, resulting in higher amplitudes of $I_{P}$. Since the values used are $\Gamma_{1}=0.20$ and $\Gamma_{2}=0.80$, the amplitude of $I_{P}$ is smaller in comparison to the shift along the current axis. On the other hand, by comparing the figures $2 \mathrm{~b}$ and 2 , we note that the amplitude of $I_{A P}$ is almost independent 

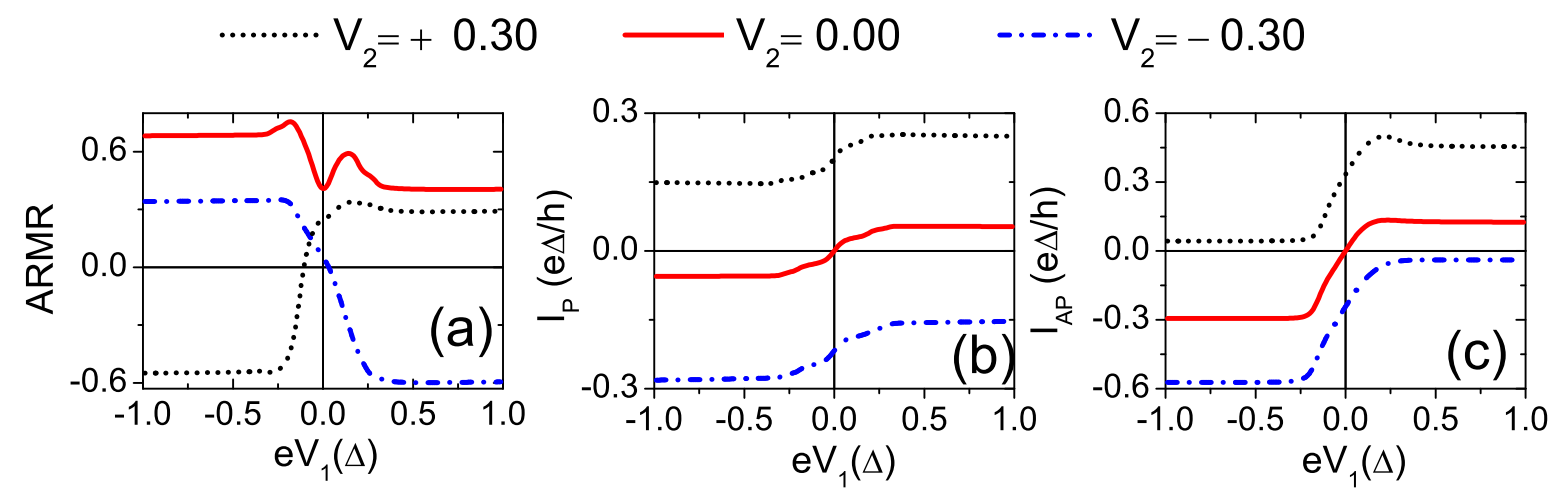

FIG. 2. (Color Online) Magnetoresistance $A R M R$ and corresponding currents $I_{P}$ and $I_{A P}$ through the system for different values of the applied bias $V_{1}$ and $V_{2}$. (a) $A R M R$. (b) $I_{P}=I(\theta=0)$. (c) $I_{A P}=I(\theta=\pi)$. Fixed parameters: $\Gamma_{1}=0.20$, $\Gamma_{2}=0.80, \Gamma_{s}=0.30, t_{a b}=0.20, V_{g a}=V_{g b}=0, \mathcal{U}=0.40, P_{1}=P_{2}=0.95, k_{B} T=0.01$. All the parameters are expressed in superconductor gap units.

on the relation between $\Gamma_{1}$ and $\Gamma_{2}$. In fact, $I_{A P}$ is carried almost through crossed ARs which picks up one up-spin electron from $F_{1}$ and another down-spin electron from $F_{2}$. Since the total current entering into the superconductor must be unpolarized, it is limited by the electrode with lower injection of electrons. The difference between these two processes (crossed and direct AR) with respect to the variations of $\Gamma_{1}$ and $\Gamma_{2}$ allows the control of the sign of $A R M R$ through external parameters.

The curves for $I_{A P}$ display some interesting features. Unlike the current $I_{P}$, the shift of $I_{A P}$ along the current axis is related to the applied bias rather than the coupling constants $\Gamma_{1}$ and $\Gamma_{2}$. In fact, the zero value of the current $I_{A P}$ is found through the condition $V_{1}=-V_{2}$ (see also Ref. 58 for one-dot case). This condition determines the shifts of the current $I_{A P}$ when the value of the bias is changed in the electrode $F_{2}$. The current $I_{A P}$ is given by the sum of the currents of each electrode, $I_{1}$ and $I_{2}$, which present the same behavior shown by the total current. This is a result of the coherence between the leads in the crossed AR. To illustrate this point, in Fig. 3 the current curves in the electrode $F_{1}$ are shown for values of the intradot interaction $\mathcal{U}$ ranging from 0 up to 0.80 . By comparing the curves of Figs. 3r and 2k, it can be noted that the amplitude of $I_{1}$ is half the amplitude of the total current showing a balance in the contribution of each ferromagnet. For all curves shown in Fig. 3, the system works as a switch when $\left|V_{1}\right| \gtrsim 0.32$ (out of the shaded region): if the bias in $F_{2}$ is changed from zero to \pm 0.30 the current through $F_{1}$ is commuted from its maximum to a value close to zero. In Fig. $2 k$, the current is reduced from 0.20 to $4.5 \times 10^{-3}$ at $V_{1}=0.60$ as $V_{2}$ is changed from +0.30 to -0.30 . This implies that the current is reduced to $2 \%$ of its maximum value. This small "leakage" current could be eliminated in the case in which the ferromagnets are fully polarized. This switching behavior of the system can be useful in practical applications since the system behaves as a transistor. The switching effect persists even for high values of the intradot inter- action as shown in Fig. 3f, for $\mathcal{U}=0.80$. However, as the interaction increases, an asymmetry in the curves with respect to the sign of $V_{1}$ emerges: the amplitude of $I_{1}$ is strongly reduced for $V_{1}>0$ but is weakly reduced for $V_{1}<0$. In curves with $V_{2}=+0.30$, there is a reduction of the current with the increase of the applied potential for $\mathcal{U}>0.20$. This effect has been studied by the authors in a previous work ${ }^{62}$ and its explanation is based on the appearance of asymmetries caused by the interaction in the local density of states (LDOS) at the QDs.

The results for the transistor based on AR depend on high values of the polarization of the electrodes since the difference between direct and crossed processes is the key for the behavior observed in this system. In addition, the step-like behavior of the current stems from the localized LDOS around the superconductor chemical potential $\left(\mu_{S}\right)$. This way, in an experimental realization of this system, a pertinent question would be if the transistor effect persists for smaller values of polarization and hopping between the QDs. The latter parameter being responsible for the resonant structure of the LDOS around $\mu_{S}$. In order to analyze these points, in Fig. 4 some curves for the current $I_{1}$ are presented for different values of the ferromagnet polarizations and hopping parameter between dots. We considered three different values of polarizations: $P_{1}=P_{2}=0.95$ (Figs. $4 \mathrm{~b}$ and $4 \mathrm{~b}$ ); $P_{1}=P_{2}=0.60$

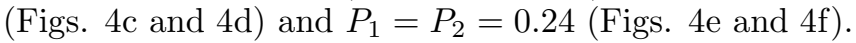

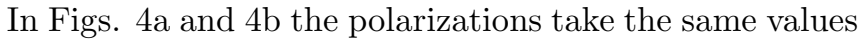
as in Figs. 22 and 3. but $t_{a b}$ is ranging from 0.05 up to 0.20 . As $t_{a b}$ is reduced from the value of 0.20 , the amplitude of the current is strongly reduced for both signs of $V_{2}$. In spite of this reduction, the dependence of $I_{1}$ on $V_{1}$ is qualitatively the same for all values of $t_{a b}$. Thus, the variation of $t_{a b}$ within this range of values preserves the behavior of the system as a transistor. In Figs. 4 c and $4 \mathrm{~d}$, the polarization is reduced to $P_{1}=P_{2}=0.6$. In this case, an important change can be observed in comparison to the curves of Figs. 近 and $4 \mathrm{~b}$ : the leakage current displays now a noticeable value which increases 

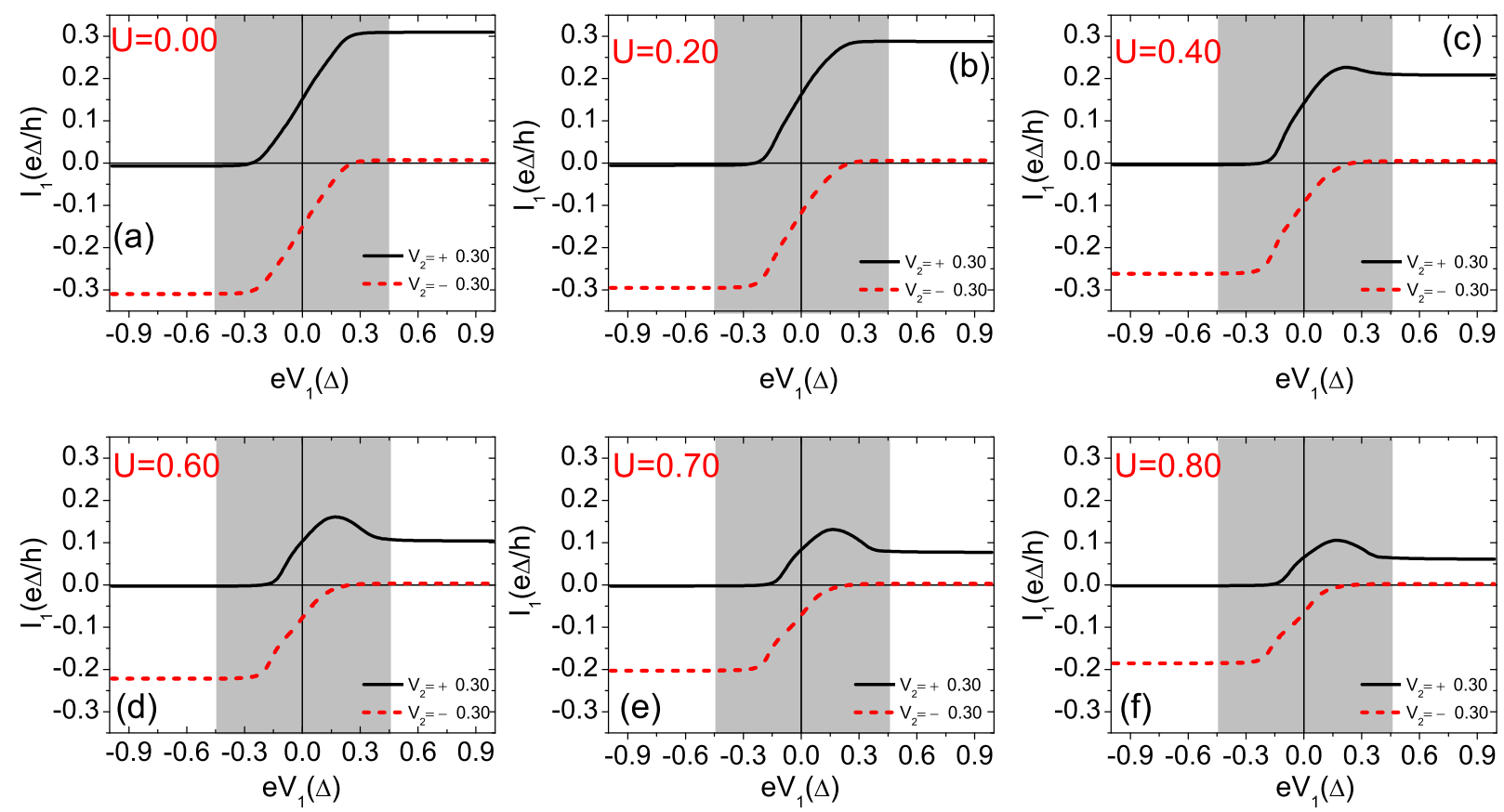

FIG. 3. (Color Online) Current through the terminal $F_{1}$ for $V_{2}=-0.30$ (red curve) and $V_{2}=0.30$ (black curve) for different values of the intradot interaction $\mathcal{U}$. Fixed parameters: $\theta=\pi, \Gamma_{1}=0.20, \Gamma_{2}=0.80, \Gamma_{s}=0.30, t_{a b}=0.20, V_{g a}=V_{g b}=0$, $P_{1}=P_{2}=0.95, k_{B} T=0.01$. All the parameters are expressed in superconductor gap units.

with the hopping parameter. For $t_{a b}=0.20$, the maximum value of the leakage current is approximately \pm 0.02 for $V_{1}= \pm 0.78$ and $V_{2}=\mp 0.3$. As the hopping parameter is reduced, the leakage current is also reduced as shown in Figs. 4t and 4 $\mathrm{d}$ in which $t_{a b}$ is changed from 0.20 to 0.05 . Even though, for all curves the maximum value of the leakage current is about $13 \%$ of the current maximum for both signs of $V_{2}$. As the polarization is further reduced, the leakage current increases as shown by the Figs. 4 and 4 ; , for $P_{1}=P_{2}=0.24$. In this case, the leakage current is about $20 \%$ of the maximum current. Hence, the switching effect of the system becomes less efficient as the polarization is reduced below $80 \%$. On the other hand, the reduction of $t_{a b}$ does not destroy the switching effect since it just reduces the current amplitudes.

In the results shown in Figs. 2 to 4, the intradot interaction has introduced a negative differential conductance on the current response. However, there are other effects which can also take place under the presence of electronic correlations at the QDs. In particular, the intradot interaction splits the up and down-spin states at each QD, with the corresponding splitting of peaks in the transmittance and differential conductance. In the case in which crossed ARs are present, the effect of $\mathcal{U}$ on the spin-degeneracy is more complex in comparison to systems with two-terminals as the one studied in Ref. 62. To illustrate this point, a comparison between the responses of the present system and the twoterminal system of Ref. 62 is shown in Fig. 5. The solid curve (black curve) corresponds to the total current through the system $F_{1}-Q D_{a}-Q D_{b}-S$ and the dotdashed curve (red curve) is the total current flowing in the $\left(F_{1}, F_{2}\right)-Q D_{a}-Q D_{b}-S$ system. For the first system, which conducts via normal ARs, we chose a not so big value of the polarization $\left(P_{1}=0.50\right)$, otherwise the current will be very small. For the two-terminal system, both polarization are close to 1 , and the current is mainly due to non-local crossed ARs. The corresponding differential conductance curves are shown in Fig. 5b. For the system with one ferromagnet, the current displays eight steps corresponding to the eight peaks of the differential conductance. Note that admixture between the QD levels with the continuum of states from the ferromagnet and the Andreev levels of the superconductor, gives rise to a four-peak structure of the LDOS at the QDs 62 . Under the presence of the intradot interaction, those four peaks are split, resulting in eight peaks in the LDOS and eight steps in the current. In contrast, only four peaks appear in the figure of the differential conductance for the system with 2 ferromagnets. To understand the difference between those responses, the key factor is to note that the polarizations have being chosen close to unity in the two-terminal system. As a result, the conduction is carried mainly by crossed ARs, since the ferromagnets are in the antiparallel configuration. The electrodes carry the current in a coherent way, i.e., one spin-up electron from $F_{1}$ and another spin-down electron from $F_{2}$ are combined as a Cooper pair in the superconductor. In Fig. 55 the transmittance curves for crossed ARs are shown. $T_{A R, 12}$ represents the transmittance for a spin-up electron of $F_{1}$ to be reflected as a spin-down hole in $F_{2}$, while $T_{A R, 21}$ 

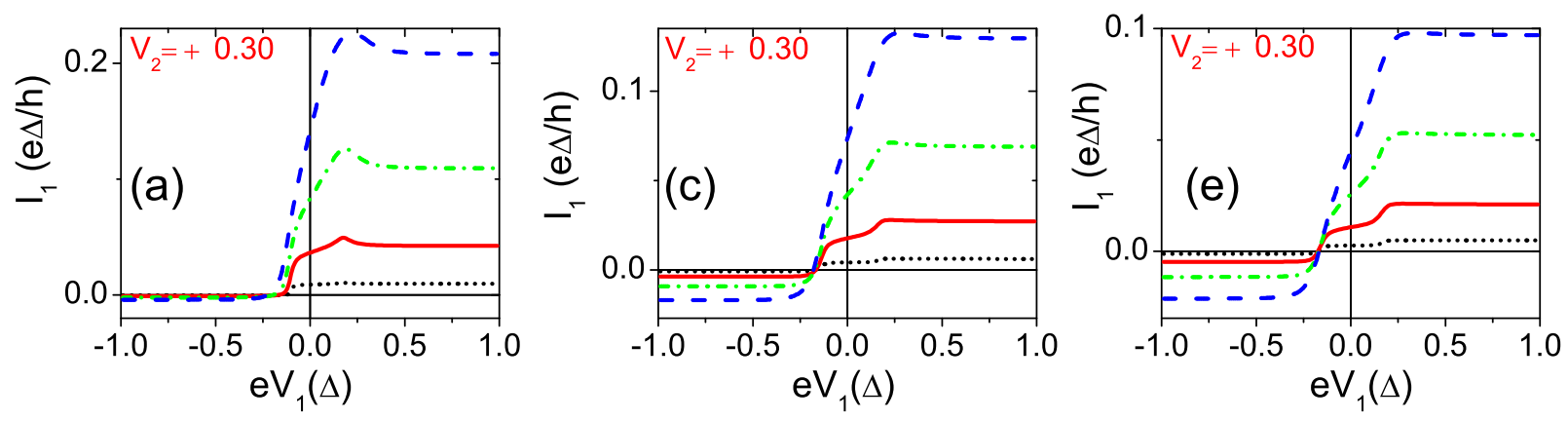

$\cdots \cdots \mathrm{t}_{\mathrm{ab}}=0.05-\mathrm{t}_{\mathrm{ab}}=0.10-\cdots \cdot \mathrm{t}_{\mathrm{ab}}=0.15--\mathrm{t}_{\mathrm{ab}}=0.20$
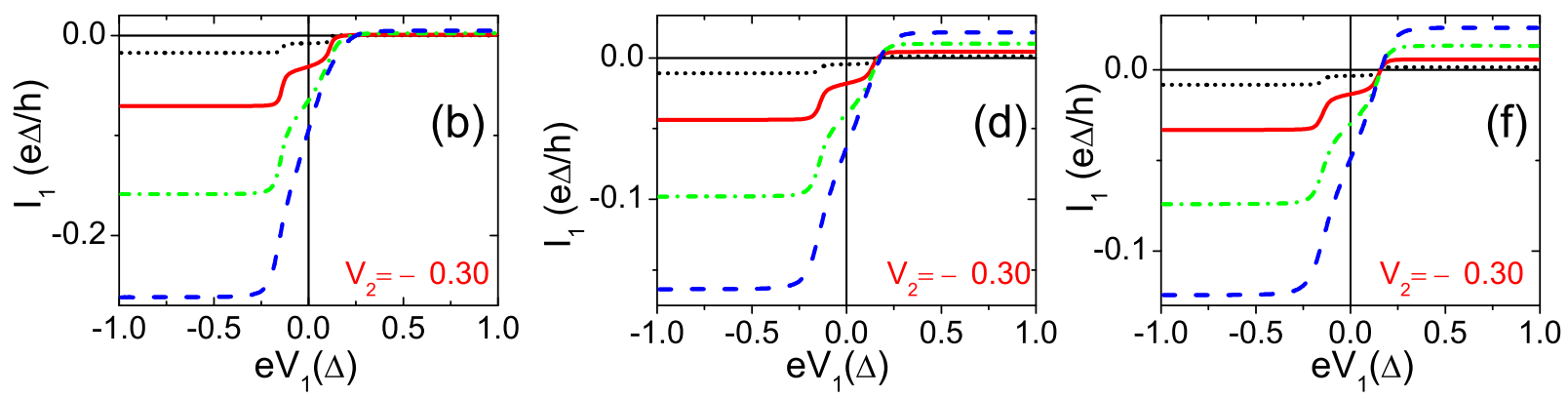

FIG. 4. (Color Online) Current through the terminal $F_{1}$ for different values of hopping parameter $t_{a b}$. Figs. (a) and (b): $P_{1}=P_{2}=0.95 ;(\mathrm{c})$ and $(\mathrm{d}): P_{1}=P_{2}=0.60 ;(\mathrm{e})$ and $(\mathrm{f}): P_{1}=P_{2}=0.24$. Fixed parameters: $\theta=\pi, \Gamma_{1}=0.20, \Gamma_{2}=0.80$, $\Gamma_{s}=0.30, \mathcal{U}=0.40$ and $k_{B} T=0.01$. All the parameters are expressed in superconductor gap units.

represents the transmittance for a spin-down electron of $F_{2}$ to be reflected as a spin-up hole in $F_{1}$. It can be noted that the curves are not symmetric with respect to the energy origin. This feature contrasts with the transmittance in the case of one ferromagnetic electrode, which is illustrated in Fig. 55d. However, the two curves displayed in (c), when combined present a symmetric character, as one can observe by locating the peaks of each spectrum. The peak labeled by 1 in $T_{A R, 12}$ and the peak $1^{\prime}$ in $T_{A R, 21}$ are located at $\omega=+0.16$ and -0.16 , respectively. The same symmetry can be observed between the other pairs of peaks. This shows us that the ferromagnets act cooperatively in the transport, circumventing the effect of $\mathcal{U}$ in breaking spin degeneracy. In fact, the interaction $\mathcal{U}$ is only responsible for the shifts of the curves with respect to the origin. However, since $T_{A R, 12}$ and $T_{A R, 21}$ are displaced symmetrically, there is no sensible effect on the current and differential conductance as shown in Figs. $5 \mathrm{~b}$ and $5 \mathrm{~b}$. In the case with one ferromagnet, there is only one transmittance curve, and the effects of the interaction can be observed by the splitting of the peaks. It is important to note that transport via $\mathrm{AR}$ requires electrons with energies disposed symmetrically with respect to the superconductor chemical potential. This is necessary in order to form Cooper pairs within the superconductor and sustain the subgap current associated with AR. Next, we consider the effect of the gate potentials on the electric transport of the system. In Fig. 6, the current through $F_{1}\left(I_{1}\right)$ is plotted in terms of the gate potentials $V_{g a}$ and $V_{g b}$ applied on the QDs $a$ and $b$, respectively. The bias in the ferromagnets are fixed at $V_{1}=V_{2}= \pm 0.30$ corresponding to the maximum value of $I_{1}$ for the curves of Fig. $4 \mathrm{~b}$ and $4 \mathrm{~b}$. In Figs. $6 \mathrm{~b}$ and $6 \mathrm{~b}$ the interaction at the QDs is zero and $I_{1}$ displays a single peak centered at $V_{g a}=V_{g b}=0$. The same behavior is observed in Fig. 6b for $V_{1}=V_{2}=-0.30$. When the interaction at the QDs is present, the current still exhibits a single peak as observed in Figs. 6r and 6d for $\mathcal{U}=0.80$. However, the peak is now located at $V_{g a}=V_{g b}=-0.18$ and its amplitude has been reduced to half of the value for $\mathcal{U}=0.0$. Therefore, under the presence of interaction at the QDs, gate voltages must be used in order to find the maximum condition for the electrical current. By the projections on the plane $V_{g b} \times V_{g a}$ in Figs. 6 $\mathrm{b}$ and $6 \mathrm{~d}$, it can be noted that the variation of $I_{1}$ is asymmetric with respect to the gate potentials. In fact, $I_{1}$ is different from zero in the entire range of $V_{g a}$ but is appreciable only within a very narrow range of $V_{g b}$. This can be explained by noting that the system is not symmetric, with each QD connected to a different electrode, and subjected to a different hybridization of quantum states. This is also reflected in the structure of the LDOS in both QDs, as shown in Fig 7, By changing the gate voltages $V_{g a}$ and $V_{g b}$, it is possible to change the QDs levels and the LDOS. In simpler systems, in which the coupling between the QDs and the electrodes is weak, the gate voltage just shifts the QDs levels with respect to the chemical potential of the electrodes. Hence, the behavior 

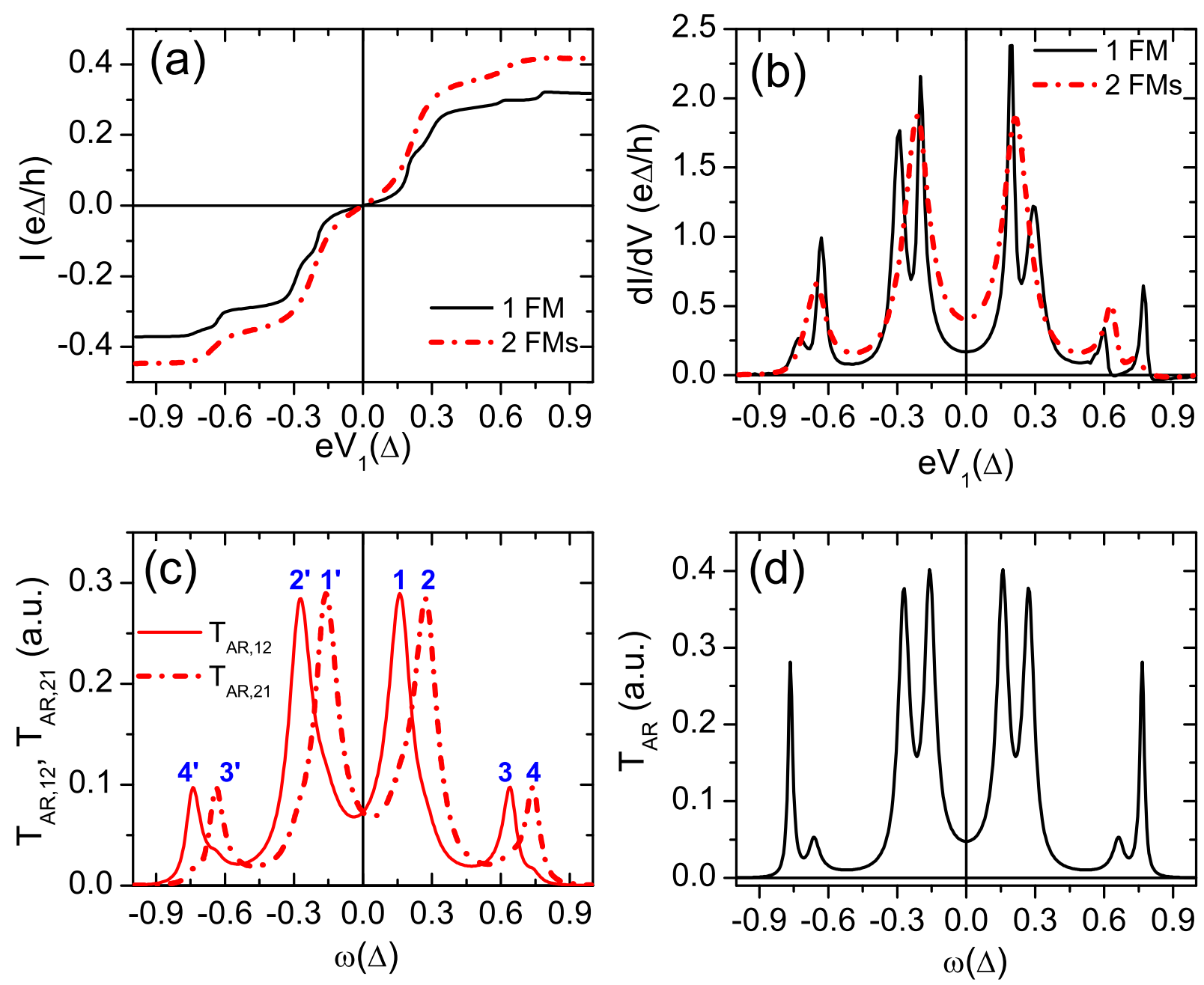

FIG. 5. (Color Online) (a) Currents through the one-terminal $F_{1}-Q D_{a}-Q D_{b}-S$ system (solid black curve) and the twoterminals $\left(F_{1}, F_{2}\right)-Q D_{a}-Q D_{b}-S$ system (red dash-dotted curve). (b) Corresponding differential conductance curves. (c) Transmittance curves for crossed AR of the two-terminal $\left(F_{1}, F_{2}\right)-Q D_{a}-Q D_{b}-S$ system. $T_{A R, 12}$ represents the transmittance for an up-spin electron of $F_{1}$ to be reflected as a down-spin hole of $F_{2} ; T_{A R, 21}$ represents the transmittance for an up-spin electron of $F_{2}$ to be reflected as a down-spin hole of $F_{1}$. (d) Transmittance curve for the one-terminal system $F_{1}-Q D_{a}-Q D_{b}-S$. Fixed parameters: $\Gamma_{1}=0.20, \Gamma_{s}=0.30, \mathcal{U}=0.90, t_{a b}=0.20, k_{B} T=0.01, V_{2}=0.30 . \Gamma_{2}=0.80$ and $P_{1}=P_{2}=0.95$ for the system with two-terminals, and $\Gamma_{2}=0$ and $P_{1}=0.50$ for the system with one ferromagnet. All the parameters are expressed in superconductor gap units.

of the LDOS can be described in a intuitive manner, being possible to relate the changes of the current directly with those of the levels of the QDs. In our example, the analysis is subtler, since the QD levels are admixed with the continuous band of the ferromagnets and the discrete Andreev levels of the superconductor. This way, the effects of the gate voltages on the LDOS are more complex to resolve. To illustrate this point, in Fig. 7 we display some curves for the LDOS of both QDs, for different values of $V_{g a}$ and $V_{g b}$. The LDOS are obtained by standard methods, from the imaginary part of elements $11(\operatorname{dot} a)$ and $33(\operatorname{dot} b)$ of the retarded Green function of these QDs. Explicit formulae can be found in Ref. 62 .

The curves correspond to the bias $V_{1}$ and $V_{2}$ equal to 0.30 meaning that only the states within the range of conduction $-0.30<\omega<+0.30$ are contributing to the transport. As the gate voltages are varied, the LDOS within this window changes, modifying the response of the system to applied voltages in $F_{1}$ and $F_{2}$. For the non-interacting case, the maximum value of the current occurs for $V_{g a}=V_{g b}=0$. The corresponding LDOS for both QDs are represented by the solid curves (black curves) in Figs. $7 \mathrm{~b}$ and $7 \mathrm{~b}$. It can be noted that the LDOS is spread over the entire range $-0.30<\omega<+0.30$ which implies that the current is carried almost over all range determined by the applied bias. For $V_{g a}=-0.90$ (blue dotted curve) and $V_{g a}=+0.90$ (red dash-dotted curve) LDOS-A exhibits a maximum at $\omega=-0.93$ and +0.93 , respectively. In the range of interest, there are two well localized peaks but with asymmetric amplitudes. 

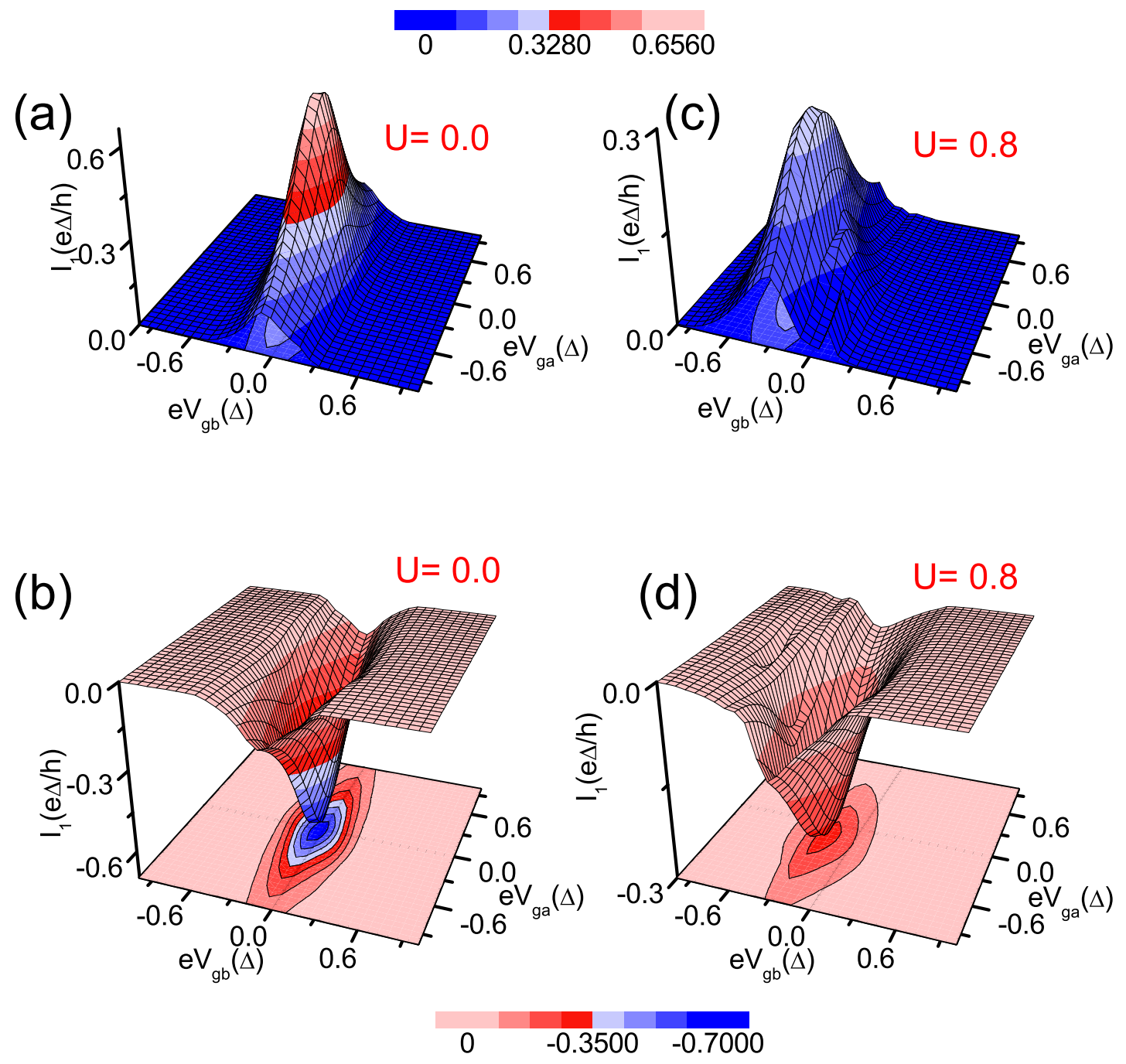

FIG. 6. (Color Online) Current through the terminal $F_{1}$ as a function of the gate voltages $V_{g a}$ and $V_{g b}$. (a) $V_{1}=V_{2}=+0.30$ and $\mathcal{U}=0$. (b) $V_{1}=V_{2}=-0.30$ and $\mathcal{U}=0$. (c) $V_{1}=V_{2}=+0.30$ and $\mathcal{U}=0.8$. (d) $V_{1}=V_{2}=-0.30$ and $\mathcal{U}=0.80$. Fixed parameters: $\theta=\pi, \Gamma_{1}=0.20, \Gamma_{2}=0.80, \Gamma_{s}=0.30, t_{a b}=0.20, P_{1}=P_{2}=0.95, k_{B} T=0.01$. All the parameters are expressed in superconductor gap units.

These two peaks also appear in the curves for LDOS-B as shown in Fig. $7 \mathrm{~b}$. However, these peaks present higher amplitudes for $V_{g a}=+0.90$, being very suppressed for $V_{g a}=-0.90$. This asymmetric pattern explains the suppression of the current for these values of the gate potentials. As pointed in our previous work ${ }^{62}$, the symmetry of the peaks in the LDOS is crucial for the transport, since states located at opposite values of the energy combine to form Cooper pairs in the superconductor. If one of these peaks is suppressed, the effective number of states participating in the conduction process is effectively reduced and the current becomes smaller. For the interacting case, shown in Figs. 75 and 7bd, the intradot interaction splits the peaks of the LDOS at both QDs. However, since some peaks are strongly suppressed, we do not see them within the scale of the graph. As an example, take the value $V_{g a}= \pm 0.90$. For the QD cou- pled to the superconductor $(\operatorname{dot} b)$, the LDOS exhibits four peaks localized inside the range $-0.30<\omega<+0.30$. However, the corresponding states for the QD coupled to the ferromagnets $(\operatorname{dot} a)$ are completely suppressed in the same range (LDOS-A displays only four small peaks around $\omega=0)$. In contrast, when $V_{g a}=-0.18$, both LDOS are appreciable inside the range of conduction, which explains the maximum value of the current shown in Figs. 6r and 6d.

The system is very sensible to variations of the gate potentials, as shown by the results in Figs. 6 and 7 . In fact, the voltage values involved are restricted to the superconductor gap, and small variations of the parameters within this range are sufficient to change the transport response of the system. See, for instance, the cases of Figs. 6c and 66d, for fixed $V_{g b}=-0.18$. Changing $V_{g a}$ from -0.18 to 0 , reduces the current to $38 \%$ of its maxi- 

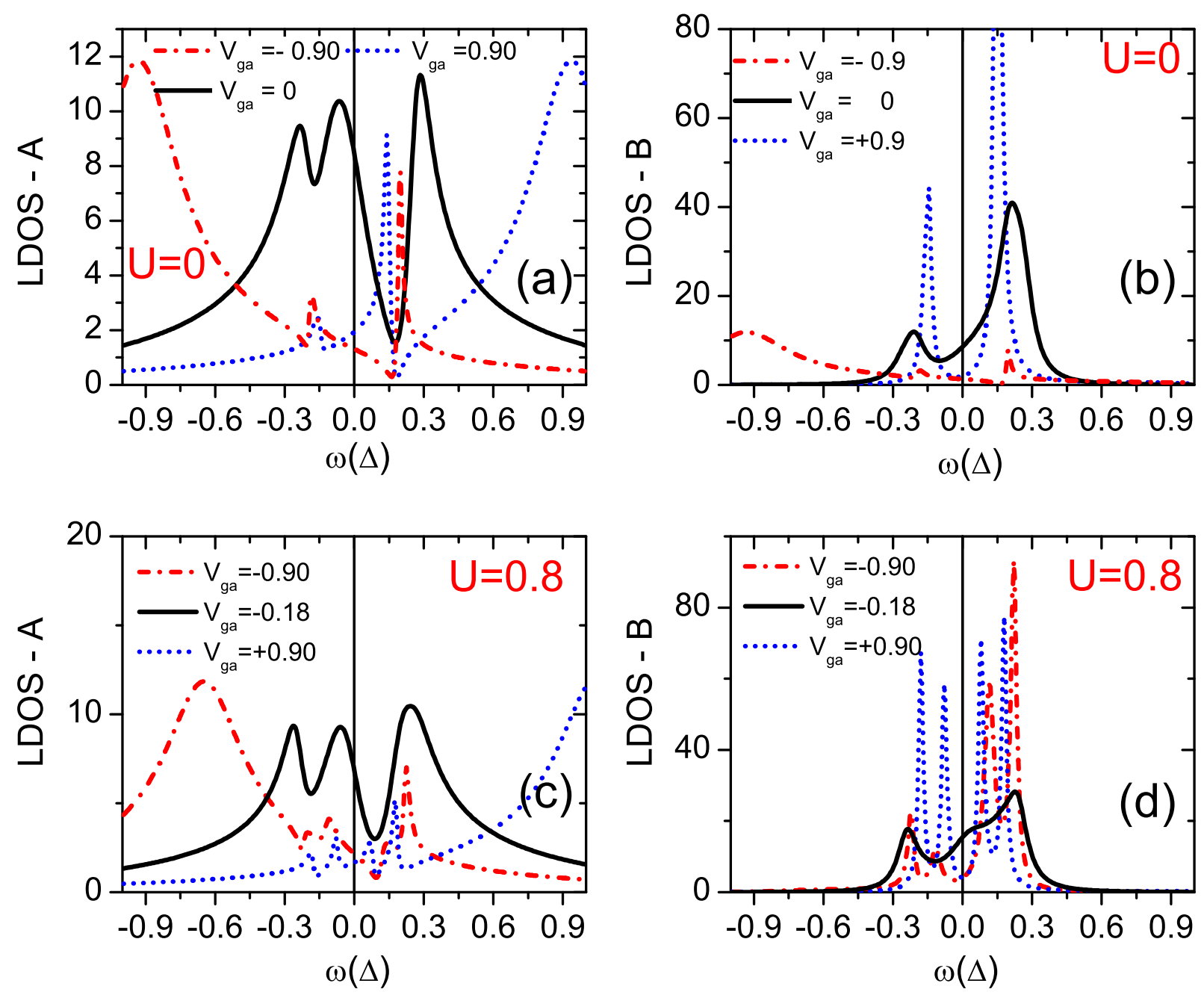

FIG. 7. (Color Online) Local density of states (LDOS) for the QDs for some values of the gate voltage $V_{g a}$. LDOS-A is the density of states of the QD connected to the ferromagnets. LDOS-B is the density of states of the second QD, connected to the superconductor. (a) LDOS-A with $V_{g b}=0$ and $U=0.0$. (b) LDOS-B with $V_{g b}=0$ and $U=0.0$. (c) LDOS-A with $V_{g b}=-0.15$ and $U=0.8$. (d) LDOS-B with $V_{g b}=-0.15$ and $U=0.8$. Fixed parameters: $\theta=\pi, \Gamma_{1}=0.20, \Gamma_{2}=0.80$, $\Gamma_{s}=0.30, t_{a b}=0.20, P_{1}=P_{2}=0.95, k_{B} T=0.01$. All the parameters are expressed in superconductor gap units.

mum value.

\section{CONCLUSION}

The combination of superconductivity with ferromagnetism in nanostructures gives rise to most interesting properties, probably useful in future technologies. In this work we have studied the magnetoresistance and the current properties of the $\left(F_{1}, F_{2}\right)-Q D_{a}-Q D_{b}-S$ hybrid system, in the case of subgap currents, when the transport is solely due to AR processes. We found that the magnetoresistance sign can be switched by applying an external potential in one of the ferromagnetic leads. In addition, the current carried by crossed ARs can also be controlled through the potential of the ferromagnets. Being a nonlocal process, crossed ARs allow control of the current in one ferromagnet, say $F_{1}$, by means of the potential applied to the other, say $F_{2}$, with the system behaving as a switch for some values of the parameters. The switching effect works better for polarization values close to unity. In fact, the leakage current in the inverse direction is completely suppressed when the ferromagnets are fully polarized. High polarizations $(>90 \%)$ values have been observed in ferromagnetic films of $\mathrm{CrO}_{2}$ by Soulen Jr. and co-workers $\underline{63}$. Some high values $(>85 \%)$ have also been reported in ferromagnetic semiconductors based on $G a M n A s \underline{64}$. This way, representative values used in our numerical calculations could be implemented in experiments. Inclusion of the intradot interaction $\mathcal{U}$ does not kill the switching effect, as shown by examples in Fig. 3. However, it is worth mentioning that our results were obtained from a mean field approximation in treating correlations at the QDs, with fluctuations be- 
ing neglected. Important effects, such as the negative differential conductance and the lifting of spin degeneracy, could be washed out by fluctuations, and we have to look for a safe ground in order to apply mean field results. Qualitatively, this domain corresponds to high polarization values and nonzero gate voltages, which strongly suppress fluctuations. However, the exact extension of the validity of the approximation used in this work can be addressed only by experiments.

The switching property shown by the $\left(F_{1}, F_{2}\right)-Q D_{a}-$ $Q D_{b}-S$ system resembles the conventional transistors used in large scale in any commercial electronic device. The future of the electronics in the nanometer domain demands devices which mimic the conventional ones, and the system presented in this work may be a contribution in this direction.

${ }^{1}$ A. F. Andreev, Sov. Phys. JETP 19, 1228 (1964).

${ }^{2}$ G. Deutscher and D. Feinberg, Appl. Phys. Lett. 76, 487 (2000). ${ }^{3}$ J. P. Morten, A. Brataas, and W. Belzig, Phys. Rev. B 74, 214510 (2006)

${ }^{4}$ C. Benjamin, Phys. Rev. B 74, 180503 (2006)

${ }^{5}$ R. Melin and S. Peysson, Phys. Rev. B 68, 174515 (2003).

${ }^{6}$ F. Giazotto, F. Taddei, F. Beltram, and R. Fazio, Phys. Rev. Lett. 97, 087001 (2006)

${ }^{7}$ G. Deutscher, Journal of Superconductivity 15, 43 (2002).

${ }^{8}$ R. Melin, Phys. Rev. B 72, 054503 (2005)

${ }^{9}$ C. Benjamin and R. Citro, Phys. Rev. B 72, 085340 (2005).

${ }^{10}$ L. Bai, Z.-Z. Zhang, L. Jiang, and F.-R. Tang, Physica E: Lowdimensional Systems and Nanostructures 43, 446 (2010).

${ }^{11}$ L. Bai, R. Zhang, and C.-L. Duan, Physica B: Condensed Matter 405, 4875 (2010).

${ }^{12} \mathrm{Z}$. Chen, B. Wang, D. Y. Xing, and J. Wang, Applied Physics Letters 85, 2553 (2004)

${ }^{13}$ X. F. Cao, Y. Shi, X. Song, S. Zhou, and H. Chen, Phys. Rev. B 70, 235341 (2004)

${ }^{14}$ J.-F. Feng and S.-J. Xiong, Phys. Rev. B 67, 045316 (2003)

${ }^{15}$ Y. Zhu, Q.-f. Sun, and T.-h. Lin, Phys. Rev. B 65, 024516 (2001)

${ }^{16}$ F. Dolcini and L. Dell'Anna, Phys. Rev. B 78, 024518 (2008)

${ }^{17}$ H.-Y. Song and S.-P. Zhou, Physics Letters A 372, 6773 (2008).

${ }^{18} \mathrm{~A}$ Brinkman and A. A. Golubov, Phys. Rev. B 74, 214512 (2006)

${ }^{19}$ C. J. Lambert, Journal of Physics-Condensed Matter 3, 6579 (1991).

${ }^{20}$ G. B. Lesovik, T. Martin, and G. Blatter, European Physical Journal B 24, 287 (2001).

${ }^{21}$ O. Sauret, T. Martin, and D. Feinberg, Phys. Rev. B 72, 024544 (2005)

${ }^{22}$ O. Sauret and D. Feinberg, Phys. Rev. Lett. 92, 106601 (2004)

${ }^{23}$ P. Simon and D. Feinberg, Phys. Rev. Lett. 97, 247207 (2006).

${ }^{24}$ G. Bignon, M. Houzet, F. Pistolesi, and F. W. J. Hekking, EPL (Europhysics Letters) 67, 110 (2004).

${ }^{25}$ Z. Y. Zeng, L. Zhou, J. Hong, and B. Li, Phys. Rev. B 74, 085312 (2006)

${ }^{26}$ W. G. van der Wiel, S. De Franceschi, J. M. Elzerman, T. Fujisawa, S. Tarucha, and L. P. Kouwenhoven, Rev. Mod. Phys. 75, 1 (2002)

${ }^{27}$ H. Liu, T. Fujisawa, H. Inokawa, Y. Ono, A. Fujiwara, and Y. Hirayama, Applied Physics Letters 92, 222104 (2008)

${ }^{28}$ G. Giavaras, J. H. Jefferson, M. Fearn, and C. J. Lambert, Phys. Rev. B 75, 085302 (2007)

${ }^{29}$ Q.-f. Sun and H. Guo, Phys. Rev. B 66, 155308 (2002).

${ }^{30}$ T. Aono and M. Eto, Phys. Rev. B 63, 125327 (2001)
${ }^{31}$ R. Aguado and D. C. Langreth, Phys. Rev. B 67, 245307 (2003)

${ }^{32}$ T. Ji, Q. Sun, and H. Guo, Phys. Rev. B 74, 233307 (2006).

${ }^{33}$ T.-S. Kim and S. Hershfield, Phys. Rev. B 63, 245326 (2001)

${ }^{34}$ Y.-X. Li, H.-Y. Choi, H.-W. Lee, and J.-J. Liu, Journal of Applied Physics 101, 103918 (2007).

${ }^{35}$ G. Giavaras, J. Wabnig, B. W. Lovett, J. H. Jefferson, and G. A. D. Briggs, Phys. Rev. B 82, 085410 (2010)

${ }^{36} \mathrm{~S}$. J. Chorley, G. Giavaras, J. Wabnig, G. A. C. Jones, C. G. Smith, G. A. D. Briggs, and M. R. Buitelaar, Phys. Rev. Lett. 106, 206801 (2011)

37Z.-Y. Zhang, Journal of Physics: Condensed Matter 18, 181 (2006).

${ }^{38}$ M. R. Buitelaar, T. Nussbaumer, and C. Schonenberger, Phys. Rev. Lett. 89, 256801 (2002)

${ }^{39}$ M. R. Buitelaar, W. Belzig, T. Nussbaumer, C. Bruder, and C. Schonenberger, Phys. Rev. Lett. 91, 057005 (2003)

${ }^{40}$ Y. Tanaka, N. Kawakami, and A. Oguri, Phys. Rev. B 78, 035444 (2008)

${ }^{41}$ Y. Tanaka, N. Kawakami, and A. Oguri, Physica E: Lowdimensional Systems and Nanostructures 40, 1618 (2008).

${ }^{42}$ J. Martinek, Y. Utsumi, H. Imamura, J. Barnaś, S. Maekawa, J. König, and G. Schön, Phys. Rev. Lett. 91, 127203 (2003)

${ }^{43}$ J. Martinek, M. Sindel, L. Borda, J. Barnaś, J. König, G. Schön, and J. von Delft, Phys. Rev. Lett. 91, 247202 (2003)

${ }^{44}$ F. S. Bergeret, A. LevyYeyati, and A. Martín-Rodero, Phys. Rev. B 74, 132505 (2006)

${ }^{45}$ D. Beckmann, H. B. Weber, and H. v. Löhneysen, Phys. Rev. Lett. 93, 197003 (2004)

${ }^{46}$ S. Russo, M. Kroug, T. M. Klapwijk, and A. F. Morpurgo, Phys. Rev. Lett. 95, 027002 (2005)

${ }^{47}$ P. Fazekas, Lecture notes on Electron Correlations and Magnetism (World Scientific, Singapore, 1999).

${ }^{48} \mathrm{~J}$. Bardeen, L. N. Cooper, and J. R. Schrieffer, Phys. Rev. 106, 162 (1957)

${ }^{49}$ F. S. Bergeret, A. F. Volkov, and K. B. Efetov, Rev. Mod. Phys. 77, 1321 (2005)

${ }^{50}$ R. S. Keizer, S. T. B. Goennenwein, T. M. Klapwijk, G. Miao, G. Xiao, and A. Gupta, Nature 439, 825 (2006).

${ }^{51} \mathrm{~F}$. Konschelle, J. Cayssol, and A. Buzdin, Phys. Rev. B 82, 180509 (2010)

${ }^{52}$ L. Trifunovic and Z. Radović, Phys. Rev. B 82, 020505 (2010).

${ }^{53} \mathrm{~L}$. Trifunovic, Z. Popović, and Z. Radović, Phys. Rev. B 84, 064511 (2011)

${ }^{54}$ L. Trifunovic, Phys. Rev. Lett. 107, 047001 (2011)

${ }^{55}$ M. Eschrig, J. Kopu, J. C. Cuevas, and G. Schön, Phys. Rev. Lett. 90, 137003 (2003)

${ }^{56}$ J. Rammer and H. Smith, Rev. Mod. Phys. 58, 323 (1986)

${ }^{57}$ Z. Y. Zeng, B. Li, and F. Claro, Phys. Rev. B 68, 115319 (2003)

${ }^{58}$ Y. Zhu, Q.-f. Sun, and T. H. Lin, Phys. Rev. B 65, 024516 (2001)

${ }^{59}$ Z. Zeng, B. Li, and F. Claro, The European Physical Journal B - Condensed Matter and Complex Systems 32, 401 (2003).

${ }^{60}$ As discussed previously, we are assuming conditions that exclude 'anomalous' AR, that is reflections with spin flip. In this case, the reflected hole flips its spin and enters the same ferromagnetic lead of the incident electron, inducing a triplet proximity effect. This local reflection will compete with the nonlocal crossed AR. However, spin-flip scattering is only possible in the presence of inhomogeities at the ferromagnets.

${ }^{61}$ K. Schwarz, Journal of Physics F: Metal Physics 16, L211 (1986)

${ }^{62}$ E. C. Siqueira and G. G. Cabrera, Phys. Rev. B 81, 094526 (2010)

${ }^{63}$ R. J. Soulen Jr., J. M. Byers, M. S. Osofsky, B. Nadgorny, T. Ambrose, S. F. Cheng, P. R. Broussard, C. T. Tanaka, J. Nowak, J. S. Moodera, A. Barry, and J. M. D. Coey, Science 282, 85 (1998).

${ }^{64}$ J. G. Braden, J. S. Parker, P. Xiong, S. H. Chun, and N. Samarth, Phys. Rev. Lett. 91, 056602 (2003) 